Table 2. Oxidation of Ascorbic ACID by Waters or Reaction Mixtures

\begin{tabular}{lcc}
\multicolumn{1}{c}{ Solution } & Rate $(\Delta O . D . / \mathrm{min})$ & $\begin{array}{c}\text { Versene concentration } \\
\text { to inhibition }\end{array}$ \\
Kitchen distilled water & 0.003 & \\
Deionized water & 0.004 & $1 \times 10^{-7}$ \\
3 $\times$ distilled water, Corning & 0.010 & $6 \times 10^{-7}$ \\
Spring water & 0.010 & $5 \times 10^{-7}$ \\
River water & 0.019 & $4 \times 10^{-8}$ \\
Weil water (tank) & 0.040 & $4 \times 10^{-6}$ \\
MgCl 0.01 M (old) & 0.055 & $3 \times 10^{-5}$ \\
Cold tap water & $0 \cdot 100$ & $3 \times 10^{-5}$ \\
Hot tap water & $0 \cdot 112$ & $7 \times 10^{-5}$ \\
Lab. tap water 0.3 ml./3 ml. & 0.204 & $3 \times 10^{-5}$
\end{tabular}

this was stopped by $2 \times 10^{-7}$ to $1 \times 10^{-6}$ molar EDTA. Sodium hydroxide at $6.7 \times 10^{-5}$ molar required $3 \times 10^{-6}$ molar ED'TA to neutralize the ascorbic acid. An old solution of 0.01 molar magnesium chloride from the shelf required $4 \times 10^{-6}$ molar EDTA. A reaction mixture for the determination of glucose-6-phosphate dehydrogenase or hexokinase contained 0.05 molar tris, 0.01 molar magnesium sulphate and 0.0011 molar glucose. Ascorbic acid was added to $6 \cdot 7 \times 10^{-5}$ molar. The absorption at $2650 \AA$ decreased at an initial rate of $0 \cdot 100$ units $/$ min. EDTA was added until the change in absorption was 0.001 units/min and the concentration was $3 \times 10^{-5}$ molar. 'This concentration of EDTA was found to be optimal for' the hexokinase reaction. The same concentration of glutathione or mercaptoethanol protected the ascorbic acid and also the enzyme reaction.

In the course of this work it was also found that glutathione does not reduce the oxidized ascorbic acid, under the conditions of the titrations, over short periods of time. Mercaptoethanol at $5 \times 10^{-4}$ molar immediately reduced about $0.06 \mu \mathrm{moles} / \mathrm{ml}$. or about 8 per cent of the ascorbic acid which had been oxidized in the presence of copper, $1 \times 10^{-6}$ molar.

We hope that this simple method of titrating contamination of water and solutions may be found useful in other laboratories.

This investigation was supported by a grant from the National Cancer Institute, U.S. Public Health Service.

Department of Animal Biology,

School of Veterinary Medicine,

University of Pernsylvania,

Philadelphia.

\section{Hydrogen Bonding of Water in Substances containing the Amide Group}

$I_{T}$ is generally accepted that the main groups which attract water in the proteins keratin and silk are the amide (NHCO) groups. The partial molar heat of absorption $(\Delta H)$ of water at zero water content is $-3 \mathrm{kcal} / \mathrm{mole}$ referred to liquid water, and this has been considered to represent the excess strength of two $\mathrm{OH}-\mathrm{O}=\mathrm{C}$ hydrogen bonds relative to two $\mathrm{OH}-\mathrm{O}-\mathrm{H}$ bonds in liquid water. Recent work has shown that although other polymers containing the amide group, such as polyvinyl acetamide and polyvinyl butyramide, give a similar result, corresponding simple liquid amides such as $N$-methyl acetamide and $N$-n-butyl acetamide give values of $\Delta H$ about $-1 \mathrm{kcal} / \mathrm{mole}$. This would appoar to bo a property common to many polar polymers and related "monomers".

The phenomenon has been further investigated by infra-red spectroscopy. The fundamental hydroxyl stretching frequency of the water absorbed by the compounds mentioned has been recorded, and the spectrum due to the absorbato eliminated using a double beam technique.

The following facts were established

(1) In most cases, with both polymers and simple amides, the hydroxyl stretching vibration frequencies of the water shifted towards lower frequencies as the amount of water present increased, and at high water contents approached the frequency found for liquid water.
(2) At low water contents (about 5 per cent) there were differences in the hydroxyl vibration frequency among the various compounds, but it was found that the frequencies in a given polymer and its corresponding model amide were almost identical. For example, the peak frequency in polyvinyl butyramide was about $3,440 \mathrm{~cm}^{-1}$, in $N$ - $n$-butyl acetamide about $3,420 \mathrm{~cm}^{-1}$; in polyvinyl $N$-methyl acetamide the frequency was about $3,490 \mathrm{~cm}^{-1}$, in $N: N$ dimethyl acetamide about $3,480 \mathrm{~cm}^{-1}$.

(3) There was no sign in any of the spectra of any band appearing in the $3,700-3,600 \mathrm{~cm}^{-1}$ region which could be cited as evidence for the non-bonding of some of the hydroxyl groups of water in solution in model amides.

It would appear from this evidence that the hydrogen bonding is identical in the polyamides and in the corresponding model compounds, on the assumption that the strength of a hydrogen bond is related to the fundamental hydrogen stretching frequency. If this is correct, then another reason must bo found for the increased values of $\Delta H$ in polymer-water systems. One possible explanation may be that put forward by Barrer et al. ${ }^{2}$ to explain the high negative heats of absorption of hydrocarbons by ethyl cellulose. He postulated the prosence of molecular cavities in the polymer in which the absorbed molecules may be accommodated with minimum distortion of the polymer lattice.

Another curious phenomenon emerges. The mixing of water with these compounds is exothermic, and it would be expected that the water would therefore be more strongly hydrogen bonded to the amides after mixing than to other water molecules beforo mixing. The frequency of the hydroxyl vibration in pure liquid water is about $3,380 \mathrm{~cm}^{-1}$, however, whereas that for water in the amides is about $3,450 \mathrm{~cm}^{-1}$, indicating that the bonding to the amides is, in fact, weaker than that in pure water. Dinkard and Kivelson ${ }^{3}$ carried out a nuclear magnetic resonance study of water and methanol in acetone and dimethyl sulphoxide and found a similar difficulty in correlating the hydroxyl proton resonance with the results of heats of mixing experiments. They found that the shifts of both hydroxyl proton resonance and infrared hydroxyl stretching frequency indicated a decrease of hydrogen bond strength with increasing dilution, while the results of heats of mixing experiments suggest otherwise. A preliminary nuclear magnetic resonance investigation of water in $N: N$-dimethyl acctamide compared with pure water has confirmed our infra-red spectroscopic findings; an increase in water concentration in the amide resulted in a shift of the proton resonance in the direction of that of pure water, that is, towards stronger hydrogen bonding.

We thank Dr. D. W. Jones, of the Bradford Institute of Technology, for recording the nuclear magnetic resonance spectra.

$$
\begin{aligned}
& \text { F. WOOT) } \\
& \text { D. K. RAMSDFN } \\
& \text { G. KrNG }
\end{aligned}
$$

Wool Industries Research Association,

Torridon, Headingley I,ane,

Leeds.

1 Ramsden, D. K., thesis, Univ. Leeds (1965).

- Barrer, R. M., Barrie, J. A., and Slater, J., J. Polymer Sci.. 27, 177 (19.5x).

${ }^{3}$ Dinkard, W., and Kivelson, D., J. Phys. Chem., 62, 1494 (1958).

\section{BIOCHEMISTRY}

\section{$\beta$-Carotene as a Photoreductone for Ferredoxin and Triphosphopyridine Nucleotide in vitro}

THE possibility that carotene participates in the photosynthesis of green plants has been frequently considered. Lynch and French ${ }^{1}$ showed that the photosynthetic activity is stopped if the carotene is removed from the 